\title{
Polyurethane scaffold for the treatment of partial meniscal tears. Clinical results with a minimum two-year follow-up
}

\author{
PAOLO BULGHERONI ${ }^{1}$, ERICA BULGHERONI ${ }^{1}$, GIANMARCO REGAZZOLA ${ }^{1}$ \\ CLAUDIO MAZZOLA²
}

${ }^{1}$ Department of Biotechnology and Life Science, University of Insubria, Varese, Italy

${ }^{2}$ Division of Joint Surgery, Galliera Hospital, Genoa, Italy

\begin{abstract}
Purpose: to evaluate the safety and effectiveness of the polyurethane meniscal scaffold through clinical examination, MRI and arthroscopic second look, over a minimum two-year follow-up.

Methods: between 2009 and 2011, 19 patients underwent meniscal scaffold implantation in our department (medial meniscus in 16 cases lateral meniscus in two cases, and bilateral in one case). All the patients were clinically evaluated preoperatively, and at 6,12 , and 24 months after surgery using Lysholm score, Tegner score, and VAS. Ten patients were studied with MRI, and nine patients were evaluated arthroscopically.

Results: no adverse reactions to the implant were observed. The clinical scores showed a significant improvement at 6 months and increased progressively over time. On MRI studies, the implants showed a clear hyperintense signal, sometimes irregular, and the chondral surface was preserved in all cases. At arthroscopic second look in the first months after surgery, the scaffold size was unchanged and the scaffold appeared light yellowish in color and well integrated into the surrounding tissues. At arthroscopic second look at 12 and 24 months the scaffold was found to have an irregular morphology and to be slightly reduced in size.
\end{abstract}

\section{Corresponding Author:}

Paolo Bulgheroni, MD

Department of Biotechnology and Life Science, University of Insubria

Via J.H. Dunant 3, 21100 Varese, Italy

Phone: +39-0332-421310/312/325

E-mail: pbulgheroni@gmail.com
Conclusions: polyurethane meniscal scaffold is a good alternative to a collagen scaffold, but a longer followup is needed to evaluate the scaffold degradation and chondral coverage.

Level of evidence: level IV, therapeutic case series.

Key Words: meniscus, polyurethane, replacement, scaffold, tear.

\section{Introduction}

The meniscus plays a cardinal role in knee physiology, especially in joint load transmission, shock absorption, joint stability, proprioception and articular lubrication (1). A meniscal lesion therefore alters the joint homeostasis, possibly leading to articular pain and later, a knee degenerative process (2-5).

Meniscal replacement can be needed when a meniscal lesion cannot be repaired. Meniscal transplantation is a well-known technique that gives satisfactory mediumand long-term results $(6,7)$. However, it is complicated, invasive, and carries a risk of transmission of infectious diseases. For these reasons, meniscal transplantation is usually unsuitable for small meniscal lesions.

The field of bioengineering has recently seen the development of three-dimensional (3D) scaffolds that allow cell migration and the formation of new vessels in order to create meniscal-like tissue $(8,9)$. These scaffolds must be porous, with proper orientation of their fibers and cavities. Their biomechanical structure must be rigid to allow tissue regeneration, while protecting the tissue from load stress, and their absorption must be sufficiently gradual. At the same 
time, it is necessary to ensure that a scaffold and its catabolites will not damage the articular surface or cause a foreign body reaction.

Different types of scaffold are currently being researched and tested, and at present only two are used in clinical practice: CMI-Menaflex (Ivy Sports Medicine; Montvale, NJ, USA) and Actifit (Orteq; Exton, PA, USA).

The CMI-Menaflex implant was proposed in 1992 (10) and has been available for clinical use since 2000. It is made from type I collagen (isolated and purified from bovine Achilles tendon) to which glycosaminoglycans are added. It has a meniscus-like shape, is implantable arthroscopically, and is biocompatible and biodegradable. It has a microscopically porous structure that induces differentiation and proliferation of fibrocartilaginous cells, leading to the creation of meniscus-like tissue, as long as the scaffold is progressively reabsorbed (9).

Animal studies and the first implants in humans in the '90s confirmed that the CMI-Menaflex implant promotes proliferation of fibrochondrocytes and the production of a new extracellular matrix (10-12).

Medium - and long - term clinical studies published in recent years suggest that it gives good results and exerts a chondroprotective effect (13-19).

Nevertheless, collagen scaffolds are fragile during the implant procedure (20), and have shown a decrease in size on magnetic resonance (MR) and arthroscopic second look follow-up.

The Actifit is a synthetic polycaprolactone-polyurethane scaffold for meniscal regeneration recently introduced for clinical use. Its structure seems to have better mechanical properties; indeed, compared with CMIMenaflex implant, it is more resistant to surgical procedures, particularly sutures, and to loads. Its greater absorption rate also allows full tissue regeneration.

Ultrastructurally, the scaffold is $80 \%$ porous; the remaining $20 \%$ is made up of polymer with a low absorption rate. Softer polycaprolactone segments constitute $80 \%$ of the polymer, while the rest is made up of more rigid urethane. Degradation starts with hydrolysis of the polycaprolactone segments, which lasts up to five years; the polyurethane segments are removed by macrophages and giants cells or integrated into surrounding tissues $(21,22)$.

The purpose of this study was to evaluate the safety and effectiveness of the Actifit polyurethane scaffold through clinical evaluation, MRI and arthroscopic follow-up. The hypothesis of the study was that the Actifit polyurethane scaffold is safe and provide a good clinical outcome.

\section{Methods}

We evaluated a consecutive series of patients treated with Actifit and followed up for a minimum of two years (range: 24-46 months). The indications for treatment were: irreparable meniscal tears requiring excision of more than $25 \%$ of the meniscal tissue or pain after previous partial meniscectomy. In this treatment, the parameniscus and part of the anterior and posterior horn of the meniscus must be preserved; the knee must be stable and correctly aligned, or surgically treated at the same time to obtain the desired stability and alignment.

Exclusion criteria were: grade 4 chondral lesions, defined according to the Outerbridge's classification, autoimmune diseases, and age over 60 years.

The study population consisted of 19 patients (20 implants), 2 females and 17 males, with an average age of 32.8 years (range: $17-50$ years). In 16 cases the medial meniscus was treated, in 2 cases the lateral meniscus, and in 1 case the treatment was bilateral. In 6 cases, the scaffold was implanted after previous meniscectomy for irreparable meniscal tears associated with anterior cruciate ligament (ACL) reconstruction. In 13 cases, the scaffold was implanted for the treatment of knee pain after previous isolated meniscus surgery (partial meniscectomy or meniscal repair).

The implantation of the scaffold was associated with ACL reconstruction in 8 cases, ACL repair in 1 case, high tibial osteotomy for varus knee in 7 cases and femoral osteotomy for valgus knee in 1 case.

The average size of the implant was $4.3 \mathrm{~cm}$ for the medial meniscus and $3.7 \mathrm{~cm}$ for the lateral meniscus. The implantation of the scaffold was performed arthroscopically. Once the correct indication had been established, all pathological tissue was removed up to the red-red or red-white zone of the meniscus. Perforations of the parameniscus were performed to obtain adequate bleeding for tissue regeneration. The size of the meniscal defect was established using a spe- 
cial arthroscopic instrument, the scaffold was trimmed to the appropriate size and inserted into the joint where it was secured with non-absorbable sutures using the all-inside and/or in-out techniques. Vertical sutures were usually applied to secure the central part, while two horizontal sutures were used at the anterior and posterior horn of the meniscus.

The rehabilitation program required the use of an articulated knee brace locked in extension for six weeks, during which the limb was kept unloaded. During the first four weeks the brace was removed 4-6 times a day to perform passive flexion from 0 to $60^{\circ}$; this was increased to $90^{\circ}$ during the following two weeks. The brace was completely removed from the eighth week. Low-impact activities were allowed at six months, whilst patients were required to refrain from participating in high-impact sports for at least nine months.

All the subjects were evaluated before surgery, and at 6, 12, and 24 months after surgery with the Lysholm, Tegner, and VAS scales. All complications were reported. All the patients underwent a magnetic resonance (MR) of the knee before surgery, whilst only 10 underwent post-operative MR, at 12 and 24 months. Patients with an osteotomy were excluded due to the presence of a metal plate.

Morphology and signal intensity were evaluated using the scale developed by Genovese et al. (23): grade 1, complete absorption of the meniscus or marked hyperintense signal; grade 2, reduced size or irregular morphology; and grade 3, normal morphology and signal intensity. Articular cartilage was assessed according to Yulish et al. (24): grade 0, normal cartilage; grade 1, signal alteration with regular edge; grade 2 , less than $50 \%$ of lesion; grade 3, higher than $50 \%$ but lower than $100 \%$, and grade 4, full-thickness ulceration.

In 9 cases arthroscopic second look and biopsy of the scaffold-regenerated tissue were performed at a considerable interval from the original surgery without damaging the implant. Specimens were processed for histological analysis and observed under polarized light microscopy.

\section{Results}

The scores on the clinical scales showed a gradual improvement over time (Fig. 1). The Lysholm score increased from an average of 66.2 preoperatively to 86.8 at 6 months, 90.7 at 12 months, and 90.5 at 24 months after surgery. The Tegner score increased from an average of 3.8 preoperatively to 4.1 at six months, 4.9 at 12 months, and 6 at 24 months. VAS score decreased from an initial value of 6.23 to 3.07 at 6
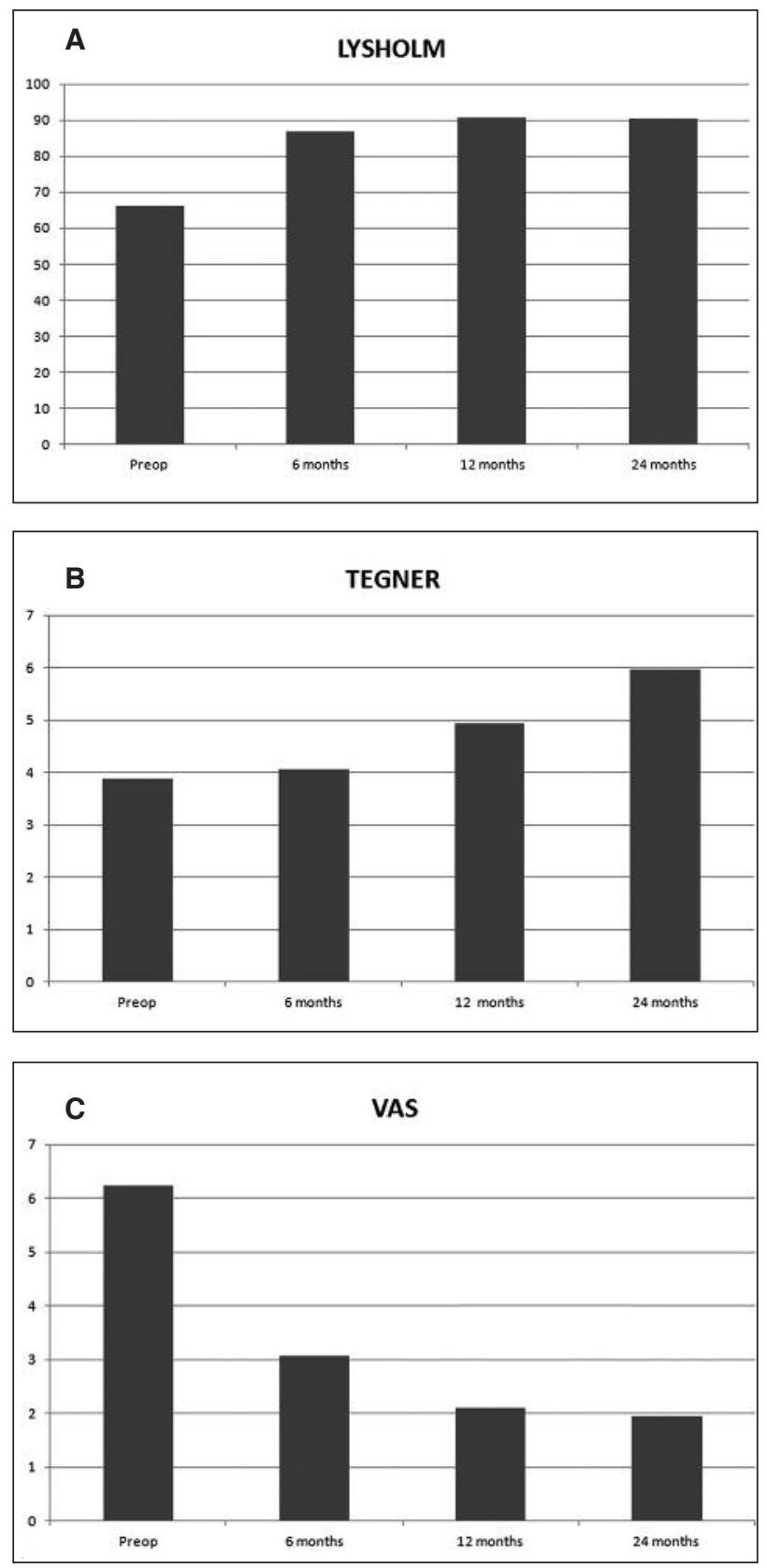

Fig. 1. Trends of Lysholm (A), Tegner (B) and VAS (C) clinical scale scores. 
months, 2.10 at 12 months, and 1.94 at 24 months. During the first 24 months the only complication was knee stiffness in a patient who underwent scaffold implantation and ACL reconstruction. It was treated with arthroscopic release which was followed by complete healing. This complication was not related to the polyurethane scaffold. Associated surgical treatments, osteotomies, and ACL reconstruction did not affect the clinical results.

On MR exams, including the evaluations performed at 24 months, the scaffolds were unchanged in shape and size but always hyperintense (Fig. 2). The mean Genovese score for morphology was 1.3 at 12 months and 1.4 at 24 months, while it was 2 for signal intensity at both time points.

The articular cartilage after surgery maintained its baseline appearance; the mean Yulish score was 1 prior to surgery and 1.1 at the 24-month follow-up.

Nine patients underwent second look arthroscopy: one underwent arthroscopic release after 4 months
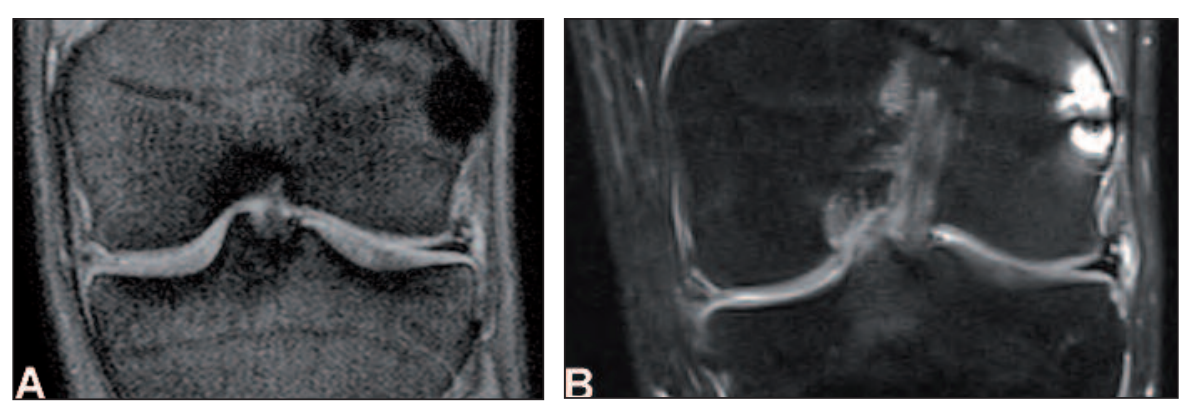

Fig. 2. MR features of the meniscal implant at 12 months (A) and 24 months (B).The implant signal, remaining hyperintense compared with that of normal meniscus, confirms its presence.
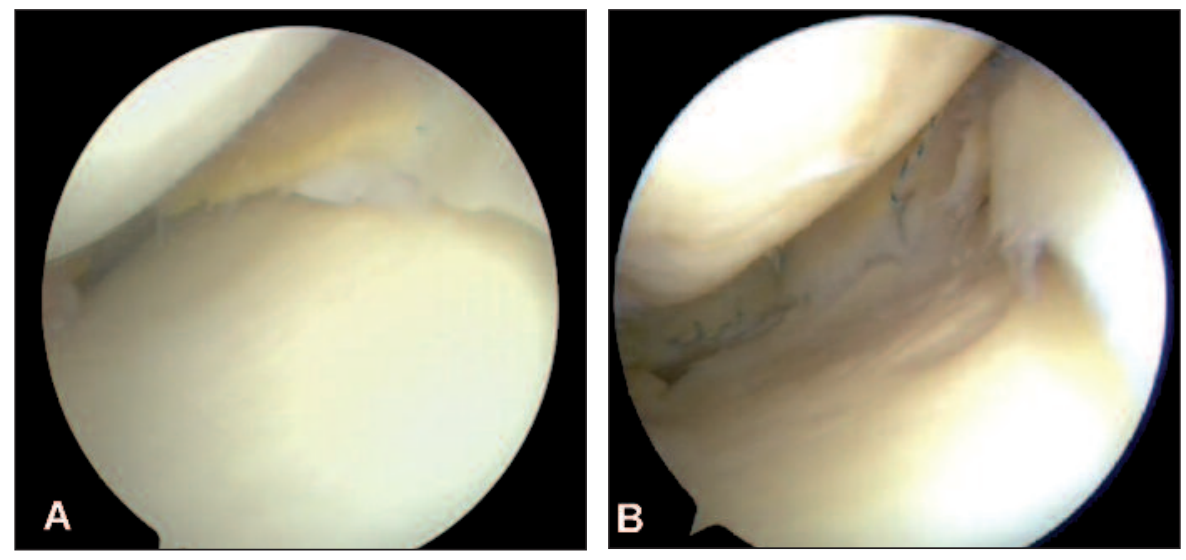

Fig. 3. Arthroscopic features of the meniscal implant. At 4 months (A) the implant has a regular shape, an unchanged size and appears well integrated. At 3 years $(B)$ it is still clearly evident but more irregular. due to joint stiffness; one after 45 months because of a knee trauma with consequent ACL injury, and the other seven patients during osteotomy plate removal between 11 and 41 months after surgery. On the arthroscopic second look performed in the first year after surgery, the implants appeared yellowish, well integrated into the surrounding tissues, only slightly reduced in size, and stable. Eighteen months after surgery the implants had a less yellowish color and often had an irregular surface; they also appeared reduced in size, particularly the inner side (Fig. 3).

Polarized light microscopy clearly identified the scaffold as birefringent. At four months after surgery new tissue had formed; this tissue had an amorphous and heterogeneous matrix and several spindle-like fibroblastic cells or more bulging fibrochondrocyte-like cells. On the biopsies performed later the cellular component seemed to be more organized with some cells appearing swollen and active, and others already fully differentiated with a typical chondrocyte-like arrangement. Vascular formations were never observed.

\section{Discussion}

Our study, considering a minimum two-year follow-up, demonstrated the safety of the Actifit scaffold. No complications emerged that could be linked to the implant.

Clinical outcomes (Lysholm, Tegner, and VAS) showed a significant improvement at six months compared with preoperative values. The results improved further until 12 months and were stable at two years. There was no difference with results recorded using CMIMenaflex implants (13).

On MR evaluation, implants were always stable, properly located, and comparable in size to the native meniscus, albeit the signal was always hyperin- 
tense. Comparing these findings with the signal intensity of the CMI-Menaflex scaffold (13), Actifit was found to be more stable over time. However, these results need to be confirmed in larger case series and with a longer follow-up.

The articular cartilage showed no sign of progression of the degenerative process. However, studies with control groups and longer follow-ups are needed to define possible chondroprotective effects.

At 18-month second look arthroscopy, the scaffold showed a constant size, good integration with the meniscal residue, and was yellowish in color, likely due to the polyurethane coming into contact with fatty acids contained in the tissues, which can cause oxidation and deterioration of the reticular structure of the polyurethane.

With longer follow-up the yellowish color became less evident and the inner side of the meniscus scaffold was often found to be irregular and reduced in size, due to the scaffold reabsorption process.

Biopsies were always taken from the free edge of the complex implant-regenerated tissue in the same area used by Verdonk for sampling (25), because this is the last part of the implant to be colonized by growing tissue and has been found to allow effective evaluation. Using polarized light, the structure of the scaffold was always recognizable; the scaffold was still evident at the latest biopsy performed (45 months after surgery). The porosity of the scaffold seemed to be filled with an amorphous and heterogeneous matrix with spindle-like and oval fibrochondrocyte-like cells. The cellular elements appeared to be better organized in biopsy specimens taken after 12 months.

There has recently been considerable interest in the use of bioengineered tissues for the treatment of partial meniscal lesions, and the literature contains various papers about collagen scaffolds, especially the CMI-Menaflex, which has been available since 2000 (9-19). All publications with a minimum 10-year follow up demonstrated significant clinical improvements (5-7). Rodkey et al. (15), in a large prospective randomized study, compared patients treated with CMI with patients undergoing partial meniscectomy; they confirmed the results reported by other authors, achieving good results in patients with chronic lesions who had previously undergone surgery on the meni- scus. MRI studies (16-19) were often characterized by a hyperintense signal and irregular surface of the meniscus, but rarely showed a progressive degenerative process of the cartilage. Our experience with arthroscopic second look often confirmed the finding of small and irregularly shaped implants (13). Histological studies on specimens showed fragments of the scaffold; only in the biopsy performed at five years was the scaffold seen to be completely reabsorbed. The regenerated tissue matures progressively and contains more cells than normal meniscal tissue. The absence of phagocytes and macrophages confirms the biocompatibility of the CMI. Some vascular formations, usually not present in meniscal tissue, have been reported (13).

Experience with polyurethane meniscal substitutes is less extensive, as these were introduced into clinical practice only in recent years. Verdonk et al. published the first results, which came from a European multicenter study on 52 patients treated with a polyurethane scaffold for partial meniscal defects $(25,26)$. In the first report (25), they evaluated tissue regeneration with "dynamic contrast enhanced" MRI performed three months after surgery and arthroscopic second look with biopsy at 12 months in 44 patients. MRI tissue regeneration evaluation was confirmed by histological findings, demonstrating a good scaffold integration and meniscal-like tissue growth. Subsequently, the same authors, reporting the clinical results recorded in the same patients after a minimum two years' follow-up, highlighted a clear increase in all the clinical outcome scores (26). One patient, although asymptomatic, underwent removal of the implant, which had not integrated with surrounding tissues. Joint cartilage appearance was stable or improved compared to MRI performed before surgery.

Efe et al. (20) reported short-term preliminary results in 10 patients treated with a polyurethane scaffold. Their study demonstrated a significant improvement in KOOS and KSS scores at 6 months, which remained unchanged until 12 months. MRI showed an improved scaffold morphology and cartilage preservation.

Spencer et al. (27) showed good results in 23 patients treated with a CMI or Actifit scaffold and followed up for an average of 19.1 months. Kon et al. (28) reported the results, at two-year follow-up, of 18 patients undergoing polyurethane scaffold implantation (13 medial 
and 5 lateral) associated with other surgical treatments such as osteotomies, ligament reconstruction, lateral release and cartilage lesion repair. The patients were evaluated using IKDC and Tegner scales and MRI. Clinical scores were significantly improved and MRI demonstrated a hyperintense signal in 10 of 16 cases.

Our study is limited by the fact that we observed a small and heterogeneous group of patients; furthermore, we considered only a short-term follow up. The absence of a control group is a further limitation. Although the clinical results were similar to those we previously reported with a collagen scaffold (13), further evaluation and a long-term follow-up are necessary to evaluate cartilage preservation and the synthetic scaffold reabsorption process.

In conclusion, synthetic polyurethane scaffold implantation was found to be a safe and effective treatment of meniscal lesions, two years after surgery. The product can be considered as an alternative to the collagen scaffold, even considering that longer follow-up studies are required to evaluate the implant reabsorption process, and the quality of the regenerated tissue.

\section{References}

1. Makris EA, Hadidi P, Athanasiou KA. The knee meniscus: structure-function, pathophysiology, current repair techniques, and prospects for regeneration. Biomaterials 2011; 32:7411-7431.

2. Fairbank TJ. Knee joint changes after meniscectomy. J Bone Joint Surg Br 1948; 30:664-670.

3. Tapper EM, Hoover NW. Late results after meniscectomy. J Bone Joint Surg Am 1969; 51:517-526.

4. Krause WR, Pope MH, Johnson RJ, et al. Mechanical changes in the knee after meniscectomy. J Bone Joint Surg Am 1976; 58:599-604.

5. Allen PR, Denham RA, Swan AV. Late degenerative changes after meniscectomy. Factors affecting the knee after operation. J Bone Joint Surg Br 1984; 66:666-671.

6. Verdonk PC, Versraete KL, Almqvist KF, et al. Meniscal allograft transplantation: long-term clinical results with radiological and magnetic resonance imaging correlation. Knee Surg Sports Traumatol Arthrosc 2006; 14:694-706.

7. Kuhn JE, Wojtys EM. Allograft meniscus transplantation. Clin Sports Med 1996; 15:537-536.

8. Buma P, Ramrattan NN, van Tienen TG, et al. Tissue engineering of the meniscus. Biomaterials 2004; 25:1523-1532.

9. Reguzzoni M, Manelli A, Ronga $\mathrm{M}$, et al. Histology and ultrastructure of a tissue-engineered collagen meniscus before and after implantation. J Biomed Mater Res B Appl Biomater 2005; 74:808-816.

10. Stone KR, Steadman JR, Rodkey WG, et al. Regeneration of meniscal cartilage with use of a collagen scaffold. Analysis of preliminary data. J Bone Joint Surg Am 1997; 79:1770-1777.
11. Stone KR, Rodkey WG, Webber RJ, et al. Meniscal regeneration with copolymeric collagen scaffolds. In vitro and in vivo studies evaluated clinically, histologically and biochemically. Am J Sports Med 1992; 20:104-111.

12. Rodkey WG, Steadman JR, Li ST. A clinical study of collagen meniscus implants to restore the injured meniscus. Clin Orthop Relat Res 1999; 367 Suppl:S281-S292.

13. Bulgheroni P, Murena L, Ratti C, et al. Follow-up of collagen meniscus implant patients: clinical, radiological and magnetic resonance imaging results at 5 years. Knee 2010; 17:224-229.

14. Steadman JR, Rodkey WG. Tissue-engineered collagen meniscus implants: 5- to 6-year feasibility study results. Arthroscopy 2005; 21:515-525.

15. Rodkey WG, DeHaven KE, Montgomery WH 3rd, et al. Comparison of the collagen meniscus implant with partial meniscectomy. A prospective randomized trial. J Bone Joint Surg Am 2008; 90:1413-1426.

16. Zaffagnini S, Giordano G, Vascellari A, et al. Arthroscopic collagen meniscus implant results at 6 to 8 years follow up. Knee Surg Sports Traumatol Arthrosc 2007; 15:175-183.

17. Zaffagnini S, Marcheggiani Muccioli GM, Lopomo N, et al. Prospective long-term outcomes of the medial collagen meniscus implant versus partial medial meniscectomy: a minimum 10-year follow-up study. Am J Sports Med 2011; 39: 977-985.

18. Monllau JC, Gelber PE, Abat F, et al. Outcome after partial medial meniscus substitution with the collagen meniscal implant at a minimum of 10 years' follow-up. Arthroscopy 2011; 27:933-943.

19. Bulgheroni P, Bulgheroni E, Vulcano E, et al. Collagen meniscus implant. A prospective study with a minimum 10 years follow-up. Journal of Orthopedics 2012; 4:117-122.

20. Efe T, Getgood A, Schofer MD, et al. The safety and shortterm efficacy of a novel polyurethane meniscal scaffold for the treatment of segmental medial meniscus deficiency. Knee Surg Sports Traumatol Arthrosc 2012; 20:1822-1830.

21. van Minnen B, van Leeuwen MB, Kors $G$, et al. In vivo resorption of a biodegradable polyurethane foam, based on 1,4-butanediisocyanate: a three-year subcutaneous implantation study. J Biomed Mater Res A 2008; 85:972-982.

22. Zuidema J, van Minnen B, Span MM, et al. In vitro degradation of a biodegradable polyurethane foam, based on 1,4-butanediisocyanate: a three-year study at physiological and elevated temperature. J Biomed Mater Res A 2009; 90:920-930.

23. Genovese E, Angeretti MG, Ronga M, et al. Follow-up of collagen meniscus implants by MRI. Radiol Med 2007; 112:1036-1048.

24. Yulish BS, Montanez J, Goodfellow DB, et al. Chondromalacia patellae: assessment with MR imaging. Radiology 1987; 164:763-766.

25. Verdonk R, Verdonk P, Huysse W, et al. Tissue ingrowth after implantation of a novel, biodegradable polyurethane scaffold for treatment of partial meniscal lesions. Am J Sport Med 2011; 39:774-782.

26. Verdonk P, Beaufils P, Bellemans J, et al. Successful treatment of painful irreparable partial meniscal defects with a polyurethane scaffold: two-year safety and clinical outcomes. Am J Sport Med 2012; 40:844-853.

27. Spencer SJ, Saithna A, Carmont MR, et al. Meniscal scaffolds: early experience and review of the literature. Knee 2012; 19:760-765.

28. Kon E, Filardo G, Zaffagnini S, et al. Biodegradable polyurethane meniscal scaffold for isolated partial lesion or as combined procedure for knees with multiple comorbidities: clinical results at two years. Knee Surg Sports Traumatol Arthrosc 2014; 22:128-134. 